Fluctuation and Noise Letters

(C) World Scientific Publishing Company

\title{
Origin of Shot Noise in Mesoscopic Cavities
}

\author{
M. MACUCCI, P. MARCONCINI \\ Dipartimento di Ingegneria dell'Informazione, Università di Pisa, Via Caruso 16, \\ Pisa, I-56122, Italy \\ macucci@mercurio.iet.unipi.it \\ Received (received date) \\ Revised (revised date)
}

\begin{abstract}
We discuss several aspects of shot noise suppression in mesoscopic cavities, focusing on the so called "quantum to classical" crossover that can be induced by an increase of the width of the constrictions defining the cavity, an increase in the energy of the injected electrons or the application of a magnetic field. After reviewing the relevant literature, we present some results of our numerical simulations, and point out an alternative explanation of the observed shot noise suppression and the reasons why several statements that can be found in the literature are debatable. Overall, we point out how shot noise behavior in mesoscopic cavities can be explained without any need for classically chaotic dynamics and only on the basis of quantum chaos resulting from diffraction at the constrictions.
\end{abstract}

\section{Introduction}

The topic of shot noise and its suppression in mesoscopic cavities has received very significant attention in the last 20 years. A very large body of literature exists, originating from early theoretical $[1,2]$ and experimental $[3,4]$ investigations of chaos in quantum dots. The first and seminal paper specifically on the noise behavior of mesoscopic cavities was published in 1994 by Jalabert et al. [5], in which, based on random matrix theory, a suppression of shot noise down to $1 / 4$ of the value given by Schottky's theorem [6] was predicted for a symmetric cavity, defined as a ballistic region connected through small constrictions to two reservoirs. The same result was then been obtained with several different approaches, both quantum and classical [7-9] and making rather different assumptions. All of these models have the presence of chaos in common, although, as we will discuss in the following, assumed as originating from different sources. The shot noise suppression by a factor $1 / 4$ was then experimentally demonstrated by Oberholzer et al. [10] for a cavity whose width was defined by etching, while the constrictions were obtained by means of quantum point contacts. A remarkable interpretation of shot noise suppression in chaotic cavities was formulated by Agam, Aleiner and Larkin [11], who introduced the concept of chaos resulting from multiple diffraction within the cavity, which occurs if the dwell time of a particle in the cavity is longer than the typical time over which 
trajectories significantly diverge as a result of diffraction, known as the Ehrenfest time $\tau_{E}$. Agam et al. also introduced a view of the transition between the regime dominated by diffraction and characterized by a shot noise suppression factor (ratio of the shot noise power spectral density to that predicted by Schottky's theorem, usually known also as "Fano factor") of $1 / 4$ to a regime without diffraction, and therefore noiseless, as a transition between a "quantum" and a "classical" behavior.

This distinction between a "classical" and a "quantum" behavior was further developed by Oberholzer and co-workers [12], who investigated shot noise suppression in a mesoscopic cavity as a function of constriction width and of an orthogonal magnetic field. While this was a remarkable experiment, we felt that the theoretical explanation was not convincing and developed an alternative model [7], based on the comparison of characteristic length scales, such as the semiclassical cyclotron radius and the constriction width.

A further development in the understanding of shot noise in mesoscopic cavities resulted from the work of Aigner et al. [13], who performed numerical simulations of shot noise in cavities of different shape (both classically chaotic and classically regular) and with the presence of a weak disorder. In particular, they noticed (in agreement with our numerical results [7]) that the shot noise power spectral density was substantially independent of the shape of the cavity, thereby ruling out conjectures that connected the observed behavior of the Fano factor with classical chaotic trajectories. They also pointed out that the main source for chaos in these structures is from diffraction at the apertures.

Theoretical efforts were also made to understand the role of symmetries [14], the dependence of conductance and shot noise on the number of propagating modes in each lead [15], the dependence of weak localization on the Ehrenfest time [16] and on magnetic field and spin-orbit coupling [17], the effect of dephasing on transport in chaotic quantum conductors [18], and the behavior of particular structures with the simultaneous presence of chaotic and regular regions [19].

Whitney and Jacquod [20] developed a semiclassical model treating a mesoscopic cavity as the parallel of two cavities: a deterministic one with electrons characterized by a short dwell time and therefore providing no contribution to shot noise and a quantum mechanical stochastic cavity with electrons that have a dwell time longer than the Ehrenfest time and generate noise; they also showed [21] that a semiclassical model can reproduce most aspects of the results obtained with random matrix theory.

More recently, Vidal and Kanzieper [22] used a scattering matrix approach to investigate the distribution of reflection eigenvalues in cavities with leads characterized by tunnel barriers.

In the present paper we review the basic concepts of the "quantum to classical" transition that has been discussed in the literature for mesoscopic cavities and formulate an intuitive interpretation of shot noise behavior in mesoscopic cavities, also with the support of some new numerical result. 


\section{2. "Classical" and "Quantum" shot noise}

As already mentioned, it is common to find in the literature (see, e.g., Ref. [12]) a distinction between "classical" and "quantum" shot noise. The basic concept is that shot noise has two main sources: the random emission from the contacts (resulting from a finite temperature) and the non-integer transmission through the device. Landauer and Martin [24] provide a detailed discussion of these noise sources, which are summarized in their Eq. (29). In the "classical" case, in which only deterministic paths exist (with either 0 or 1 transmission), the only source of shot noise is represented by the variance of the random emission from the contacts (which drops down to zero for vanishing temperature), while the purely "quantum" case is achieved at zero temperature, and thus with no fluctuation of the charge injection from the contacts, in a device in which quantum mechanical effects lead to noninteger transmission. However, a quantum device at non zero temperature exhibits both noise from charge injection fluctuations and noise from noninteger transmission, and, more importantly, a classical device such as a vacuum tetrode or pentode exhibits partition noise [25], resulting from the random capture of electrons by the screen grid, which leads to a contribution to the noise power spectral density that has the very same form as quantum partition noise, i.e. proportional to $T(1-$ $T)$, as in the purely quantum case $[26,27]$. A similar partition noise was reported also as a result of random electron-hole recombination in the base of a bipolar transistor [25]. Therefore we believe that it is better to distinguish between injection and transmission sources of noise, rather than "classical" and "quantum".

In general, the appearance of a chaotic behavior in a classically regular domain does not even necessarily involve the deterministic/non-deterministic duality, as discussed in Ref. [28], where the authors demonstrate that, within the deterministic Bohmian representation, chaos may result from the simple superposition of two properly constructed states.

If we limit ourselves to the case of the zero temperature limit, shot noise depends only on transmission, and, in general on the transmission eigenvalues for a diagonal representation of the transmission matrix, according to Büttiker's expression [27]:

$$
S_{I}(0)=4 \frac{e^{2}}{h}|e V| \sum_{i} T_{i}\left(1-T_{i}\right),
$$

where $e$ is the electron charge, $h$ Planck's constant, $V$ the voltage applied across the device, and $T_{i}$ the $i$-th eigenvalue of the $t t^{\dagger}$ matrix, $t$ being the transmission matrix.

Let us now consider the case of a mesoscopic cavity analogous to that studied in Refs. [10,12], with a rectangular shape, defined by hard walls, and with a width $W=2 \mu \mathrm{m}$ and a length $L=5 \mu \mathrm{m}$ (while in Ref. [7] we considered the very same size as in the experiment, here we consider a smaller cavity for the purpose of reducing the size of the numerical problem). A sketch of the cavity is reported in Fig. 1. 


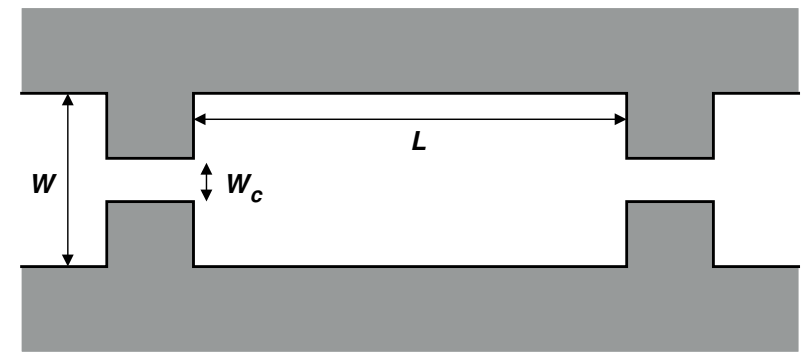

Fig. 1. Sketch of an $L$ long and $W$ wide cavity, with $W_{c}$ wide constrictions.

We have considered a rectangular, hard-wall cavity because its treatment is simpler from the numerical point of view and it has been shown that both the shape and the details of the confinement potential do not play an essential role in the behavior of the noise and of the conductance of a cavity $[7,13]$. This is a direct consequence of the fact that, as we will further discuss in the following, the quantum chaotic behavior that makes, for example, random matrix theory applicable is a consequence of diffraction at the constrictions [13], and is not related to classical chaotic dynamics, contrary to what is stated or implied by most of the literature on this subject. For all calculations, which are performed with our versions of the recursive Green's function technique $[29,30]$ and of the scattering matrix approach [31], we assume the material parameters for gallium arsenide, in particular an effective mass $m^{*}=0.067 m_{0}$, where $m_{0}$ is the free electron mass.

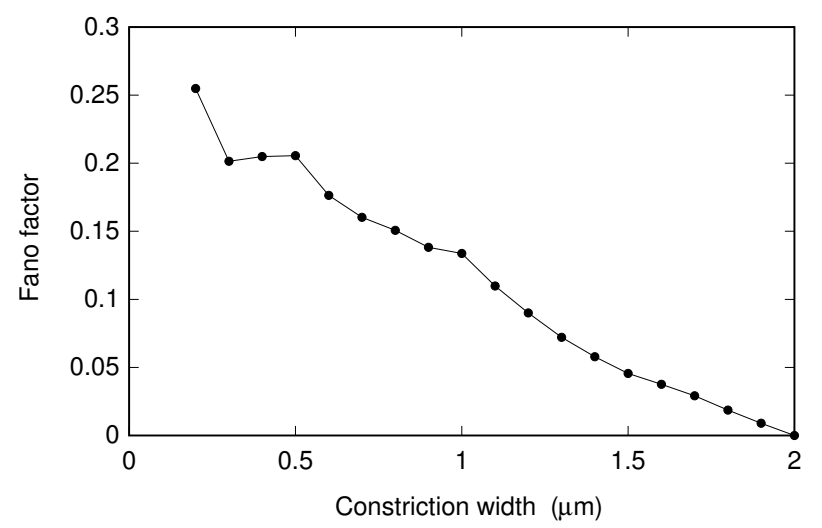

Fig. 2. Fano factor for a $5 \mu \mathrm{m}$ long and $2 \mu \mathrm{m}$ wide cavity, computed as a function of the width of its constrictions for a Fermi energy $E_{F}=9 \mathrm{meV}$, corresponding to a Fermi wavelength of $49.94 \mathrm{~nm}$

In Fig. 2 and in Fig. 3 we report the Fano factor as a function of the constriction 


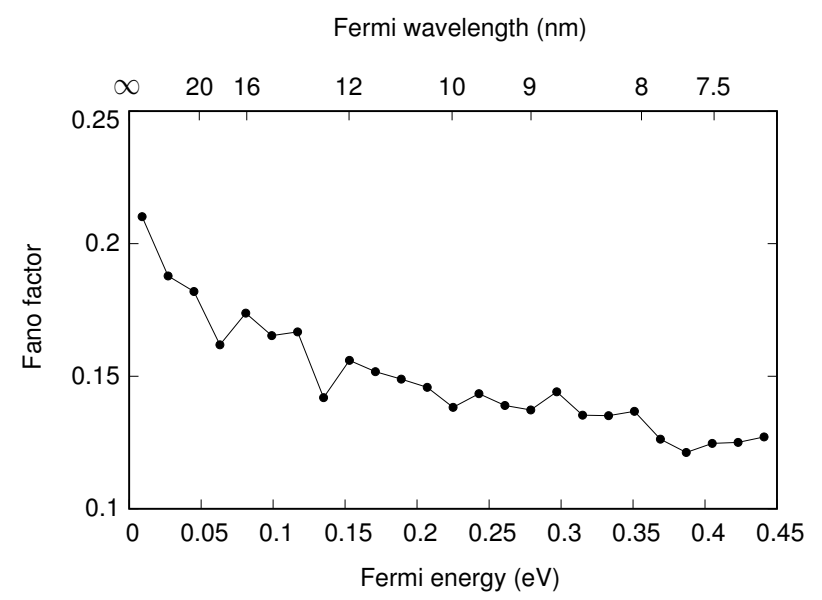

Fig. 3. Fano factor for a $5 \mu \mathrm{m}$ long and $2 \mu \mathrm{m}$ wide cavity with $500 \mathrm{~nm}$ wide constrictions, as a function of the Fermi energy and of the Fermi wavelength.

width and of the energy of the injected electrons, respectively. For Fig. 2 we have considered a constant Fermi energy $E_{f}$ of $9 \mathrm{meV}$, corresponding to the typical situation in the modulation doping layer of a GaAs/AlGaAs heterostructure, while for Fig. 3 we have considered a constant constriction width of $500 \mathrm{~nm}$. In Fig. 3 we report, on the top horizontal axis, also the Fermi wavelenght, to provide an indication of its relationship with the constriction width. While the results of Fig. 2 correspond to a realistic situation, which can in principle be implemented in an experiment (and has, at least for part of the width interval, been implemented in Ref. [12]), those of Fig. 3, instead, do not correspond to a practical situation because of the unphysically wide energy range, but are reported as a thought experiment useful to gain a better understanding of the transition to the "classical" geometrical optics regime.

Let us first discuss the data in Fig. 2: as the width of the constrictions is increased, the Fano factor, starting from a value around 1/4, decreases, dropping down to zero when the constrictions reach the same width as the main region of the cavity and therefore disappear. As previously mentioned, the diffraction occurring at the constrictions is at the origin of the quantum chaotic behavior, since diffraction of a wavepacket leads to the generation of multiple possible new trajectories. A measure of the strength of the diffraction is represented by the ratio of the constriction width to the Fermi wavelength (given by $\lambda_{f}=h / \sqrt{2 m^{*} E_{f}}$ ); at the considered Fermi energy $\lambda_{f} \approx 50 \mathrm{~nm}$, therefore strong diffraction and a Fano factor close to $1 / 4$ occur only for constriction widths up to a few hundred nanometers, while for wider constrictions a significant drop of the noise power spectral density is observed.

In Fig. 3 we see an analogous drop of the Fano factor, this time resulting from the 
fact that, as the Fermi wavelength becomes small with respect to the constriction width, a reduced diffraction regime, closer to geometrical optics, is entered, thereby reaching an increasingly "classical" behavior and a situation with reduced noise.

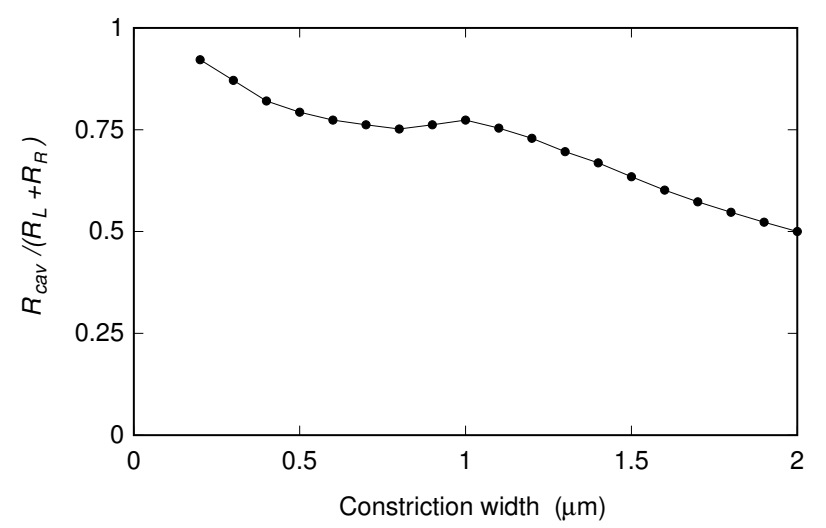

Fig. 4. Ratio of the resistance $R_{\text {cav }}$ of a $5 \mu \mathrm{m}$ long and $2 \mu \mathrm{m}$ wide cavity to the sum $R_{L}+R_{R}$ of the constriction resistances (computed from the number of modes propagating in each constriction), as a function of the constriction width, for a Fermi energy $E_{F}=9 \mathrm{meV}$, corresponding to a Fermi wavelength of $49.94 \mathrm{~nm}$.

In Fig. 4 we report the ratio of the actual resistance through the cavity to the sum of the resistances characteristic of the constrictions defining the same cavity, as a function of the constriction width. The resistances $R_{L}$ and $R_{R}$, corresponding to the resistance of the left constriction and to that of the right constriction, respectively, have been computed with the Landauer-Büttiker formula on the basis of the number of propagating modes in the corresponding contriction and assuming unitary transmission (these values have been verified also by numerical simulation of a structure with a constriction between two leads with the same width as the cavity).

According to Ref. [12] this ratio should not depend on the width of the constrictions, but we instead see that it clearly decreases as the constriction width is increased. This result is in line with those included in Ref. [7], where it has been shown to be valid also for stadium-shaped cavities. Oberholzer et al. justified their resistance data stating that this is the consequence of randomization within the cavity, which takes place both in the case of classical and of quantum chaos. We believe instead that the experimentally observed additivity of the resistances is the consequence of a non-ideality of the cavity, which is not fully ballistic because of elastic scattering from impurities or some amount of inelastic scattering. Indeed, there is a simple argument showing that the assumption of a total resistance remaining equal to the sum of the constriction resistances is not reasonable in a ballistic cavity: in the limit of constrictions as wide as the main body of the cavity, the resistance 
will be identical to that of a single constriction, therefore, as the constrictions are widened, there must be a continuous transition of the total resistance from the sum of those of the two constrictions to that of a single constriction, which is indeed reached in Fig. 4 when the width of the constrictions equals $2 \mu \mathrm{m}$, corresponding to the cavity width.

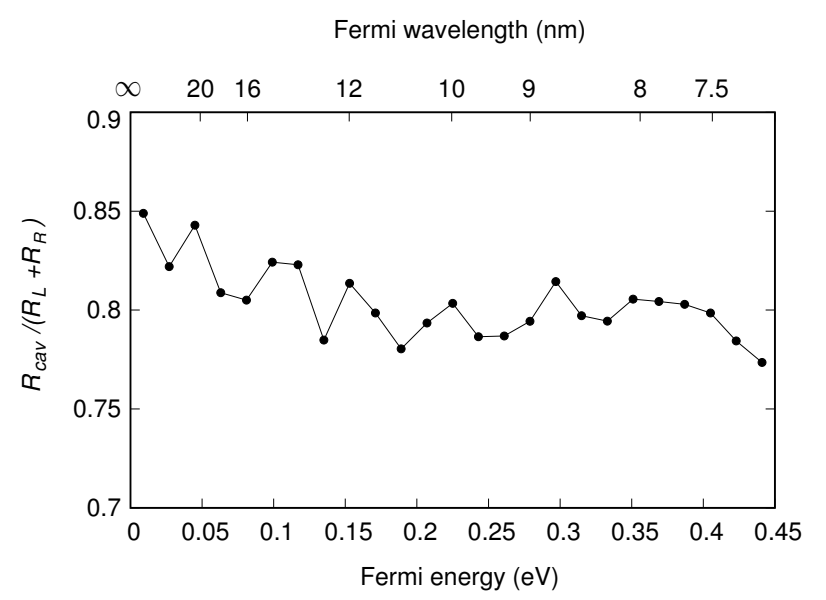

Fig. 5. Ratio between the resistance $R_{c a v}$ of a $5 \mu \mathrm{m}$ long and $2 \mu \mathrm{m}$ wide cavity and the sum $R_{L}+R_{R}$ of the constriction resistances, as a function of the Fermi energy and of the Fermi wavelength, for a constriction width of $500 \mathrm{~nm}$.

The ratio of the total resistance to the sum of the constriction resistances is shown also in Fig. 5, but in this case as a function of the energy of the injected electrons.

In several papers the presence of some form of disorder in the cavity has been invoked as a source of diffraction needed for the creation of a quantum chaotic regime or at least as contributing to the achievement of the conditions for the applicability of the results of random matrix theory and to the establishment of the transport regime characterized by a Fano factor of 1/4. For example, in Ref. [13] a relatively weak disorder is shown to raise the Fano factor to a value close to $1 / 4$ for a cavity with wide constrictions (which would otherwise exhibit a Fano factor well below 1/4). A similar result is obtained by Jacquod and Whitney [32] with a semiclassical method and by Sukhorukov and Bulashenko [33] with a path integral technique. Thus it is suggested that disorder helps in reaching the limit in which fully chaotic transport appears, but, in reality, this is just a consequence of the relatively small disorder amplitude that was considered in such studies. Indeed, we have shown [23] that as the amplitude of disorder in the cavity is increased, it contributes to a diffusive behavior typical of disordered conductors with a Fano factor greater than $1 / 4$ (1/3 for the particular case in which all requirements for 
fully diffusive transport are satisfied, a situation, however, not so common in most mesoscopic conductors [34]), prevailing over any specific cavity effect. This can be clearly seen in Fig. 6 , where the Fano factor for an $8 \mu \mathrm{m}$ wide and $5 \mu \mathrm{m}$ long cavity with randomly located hard-wall square $50 \times 50 \mathrm{~nm}^{2}$ scatterers and $800 \mathrm{~nm}$ wide constrictions is reported as a function of their number (analogous results can be obtained with realistic disorder profiles, see Ref. [23]). As the number of scatterers is increased, we notice that the Fano factor raises above $1 / 4$, without even a hint of a plateau, then reaches $1 / 3$, with a plateau, and finally rises well above such level.

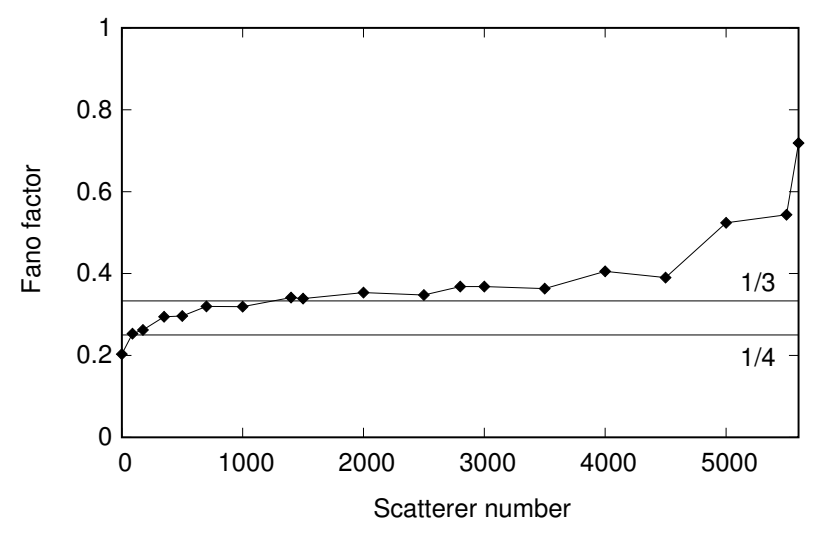

Fig. 6. Fano factor for a $5 \mu \mathrm{m}$ long and $8 \mu \mathrm{m}$ wide cavity with $800 \mathrm{~nm}$ wide constrictions, as a function of the number of randomly located square hard-wall scatterers, for a Fermi energy of $9 \mathrm{meV}$, corresponding to a Fermi wavelength of $49.94 \mathrm{~nm}$.

In the presence of a magnetic field perpendicular to the plane containing the cavity a suppression of the Fano factor was observed in Ref. [12], where an explanation was attempted on the basis of the reduced area explored by the particle within the cavity, which should lead to a reduction of the dwell time. This explanation, however, was shown not to hold [7], because changing the length of the cavity (and therefore the dwell time) the Fano factor does not vary significantly, while it is very sensitive to the ratio of the semiclassical cyclotron diameter to the constriction width [7].

As the magnetic field is increased, the cyclotron diameter $D_{C}=$ $2 \sqrt{2 m^{*} E_{f}} /(e B)$ (where $B$ is the magnetic field) decreases, edge states crawling along the walls of the cavity start forming, and cyclotron orbits start fitting within the width of the constrictions. The electron density within a cavity $8 \mu \mathrm{m}$ wide, $5 \mu \mathrm{m}$ long, and with $400 \mathrm{~nm}$ wide constrictions is shown, for a Fermi energy of $9 \mathrm{meV}$, in Fig. 7: panel a) is for no magnetic field, panel b) for an intermediate value of the magnetic field $(90 \mathrm{mT})$ and panel c) for a magnetic field of $0.84 \mathrm{~T}$, which corresponds to strongly confined edge states, leading to almost complete suppression of diffraction and therefore vanishing Fano factor. In Fig. 8 we report the behavior of 

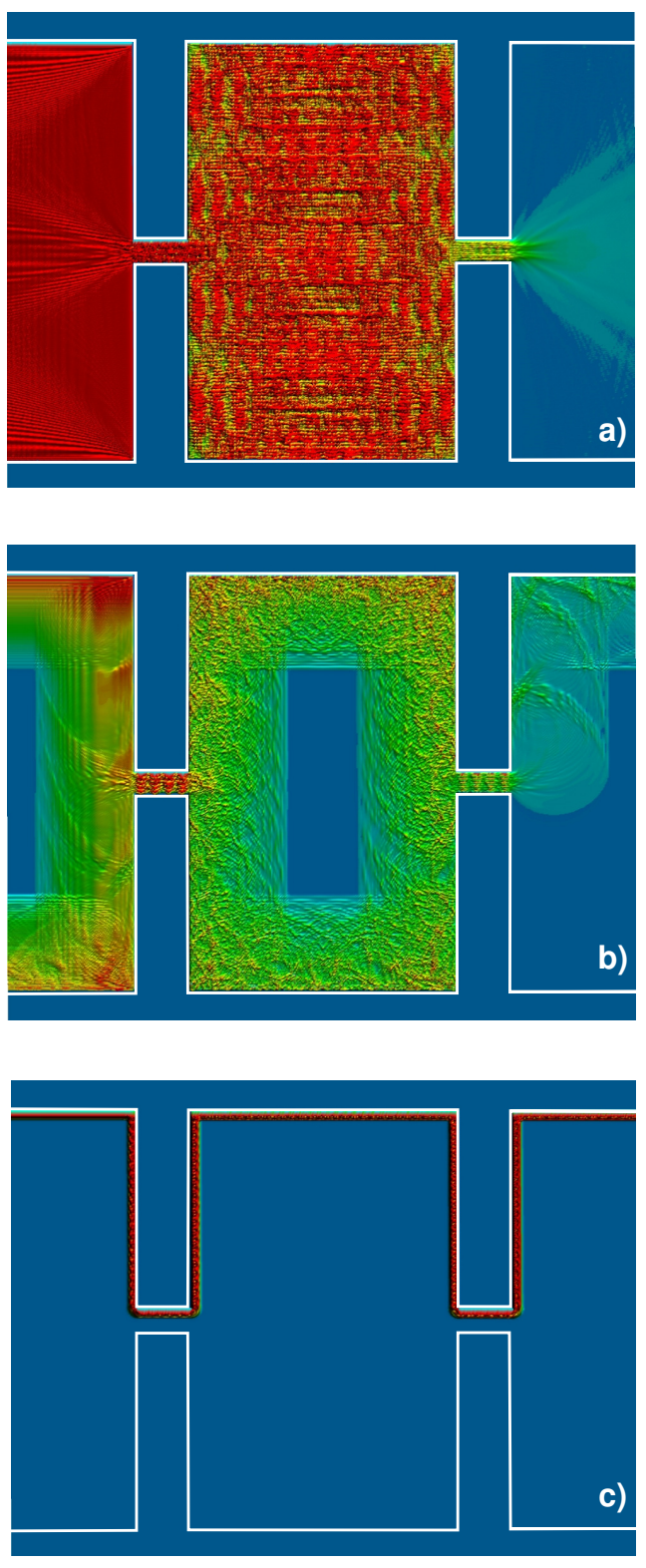

Fig. 7. Probability density for a $5 \mu \mathrm{m}$ long and $8 \mu \mathrm{m}$ wide cavity with $400 \mathrm{~nm}$ wide constrictions, computed for an orthogonal magnetic field $B$ equal to 0 (panel a), $0.09 \mathrm{~T}$ (panel b), and $0.84 \mathrm{~T}$ (panel c), for a Fermi energy of $9 \mathrm{meV}$, corresponding to a Fermi wavelength of $49.94 \mathrm{~nm}$.

the Fano factor for a cavity of the same size, but with $100 \mathrm{~nm}$ wide constrictions, as a function of the magnetic field. In this case, due to the narrower constrictions, a larger value of the magnetic field is needed to completely suppress shot noise. 
The best fit to the experimental results in Ref. [12] is obtained for a constriction width of $60 \mathrm{~nm}$ (see Ref. [7]). It is interesting to observe the behavior of the ratio of the cavity resistance to the sum of the constriction resistances reported in Ref. [12] as a function of the magnetic field: it remains somewhat constant around 1 and then starts dropping above $1 \mathrm{~T}$, quickly reaching the value 0.5 . This can be explained well on the basis of our previous interpretation: at low magnetic field the presence of elastic or inelastic scattering within the cavity effectively decouples the two constrictions, however the formation of edge states, which are protected from scattering, leads then to the behavior that we expect for a purely ballistic cavity.

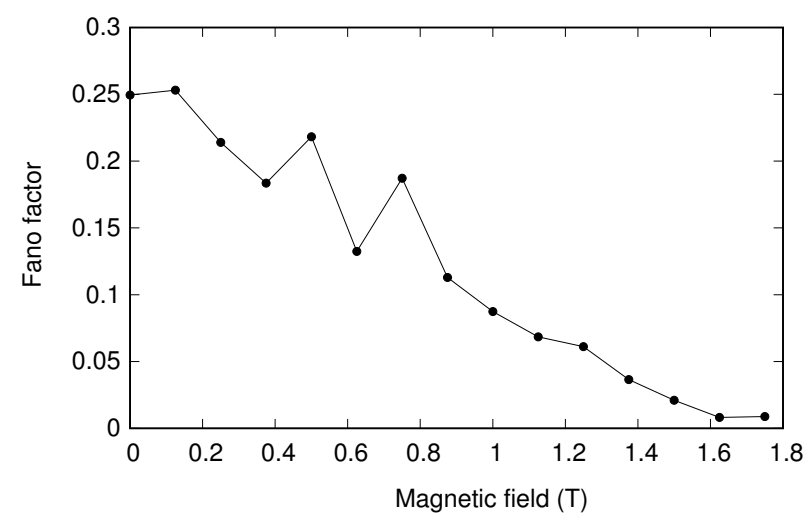

Fig. 8. Fano factor for a $5 \mu \mathrm{m}$ long and $8 \mu \mathrm{m}$ wide cavity with $100 \mathrm{~nm}$ wide constrictions, as a function of the orthogonal magnetic field $B$.

\section{Conclusion}

We have summarized the main results on shot noise in mesoscopic cavities, presenting also some new data that show how the classical limit is approached when the energy increases and the Fermi wavelength becomes much smaller than the relevant features of the confinement potential. Our main conclusions are that shot noise suppression down to $1 / 4$ of the value predicted by Schottky's theorem is the result of a quantum chaotic regime induced by wavepacket diffraction at the cavityconstriction interfaces. Contrary to what can be found in most of the literature, there is no significant contribution from classical chaotic dynamics. Indeed also the actual presence of classical chaos discussed in the early papers on quantum dots is debatable, because the confinement potential at the 2DEG (2-dimensional electron gas) level has a shape which is rather different from that of the metallic gates defining the cavity: the gate can have a classically chaotic shape, but then the effective potential confining the 2DEG may well be classically regular. Another issue we have discussed in some detail is the role of disorder in the cavity: contrary 
to several published results, it does not contribute to achieving the $1 / 4$ limit, but, rather, leads to a noise behavior typical of the diffusive regime, as long as the disorder strength is made large enough. Finally, we have discussed the issue of shot noise suppression in a cavity as a function of an orthogonal magnetic field. In this case we point out that the relevant dimensional scales are the cyclotron diameter and the constriction width: as the semiclassical cyclotron diameter decreases well below the constriction width, edge states crawl freely from one constriction to the other and noise drops, since diffraction disappears.

Overall, we can conclude that detailed numerical models are instrumental in proving or disproving conjectures that are made on the basis of simplified approaches and that one should not rely on long-term developments based on too abstract models, unless quantitative comparison to experiments or, if they are not available, with detailed numerical simulations is performed on a regular basis.

\section{Acknowledgments}

This work has been supported by the European Commission through the FP7 STREP Project GRADE under Grant 317839

\section{References}

[1] R. A. Jalabert, H. U. Baranger and A. D. Stone, Phys. Rev. Lett. 65 (1990) 2442.

[2] H. Bruus and A. D. Stone, Phys. Rev. B 50 (1994) 18275.

[3] C. M. Marcus, A. J. Rimberg, R. M. Westervelt, P. F. Hopkins and A. C. Gossard, Phys. Rev. Lett. 69 (1992) 506.

[4] A. M. Chang, H. U. Baranger, L. N. Pfeiffer and K. W. West, Phys. Rev. Lett. 73 (1994) 2111.

[5] R. A. Jalabert, J.-L. Pichard and C. W. J. Beenakker, Europhys. Lett. 27 (1994) 255.

[6] W. Schottky, Ann. Phys. (Leipzig) 57 (1918) 541.

[7] P. Marconcini, M. Macucci, G. Iannaccone, B. Pellegrini and G. Marola, Europhys. Lett. 73 (2006) 574

[8] Y. M. Blanter and E. V. Sukhorukov, Phys. Rev. Lett. 84 (2000) 1280.

[9] Ya. M. Blanter and M. Büttiker, Phys. Rep. 336 (2000) 1.

[10] S. Oberholzer, E. V. Sukhorukov, C. Strunk, C. Schönenberger, T. Heinzel and M. Holland, Phys. Rev. Lett. 86 (2001) 2114.

[11] O. Agam, I. Aleiner and A. Larkin, Phys. Rev. Lett. 85 (2000) 3153.

[12] S. Oberholzer, E. V. Sukhorukov and C. Schönenberger, Nature 415 (2002) 765.

[13] F. Aigner, S. Rotter and J. Burgdörfer, Phys. Rev. Lett. 94 (2005) 216801.

[14] V. A. Gopar, S. Rotter and H. Schomerus, Phys. Rev. B 73 (2006) 165308.

[15] D. V. Savin and H.-J. Sommers, Phys. Rev. B 73 (2006) 081307(R).

[16] P. W. Brouwer and S. Rahav, Phys. Rev. B 74 (2006) 075322.

[17] B. Beri and J. Cserti, Phys. Rev. B 75 (2007) 041308(R).

[18] R. S. Whitney, P. Jacquod and C. Petitjean, Phys. Rev. B 77 (2008) 045315.

[19] H.-S. Sim and H. Schomerus, Phys. Rev. Lett. 89 (2002) 066801.

[20] R. S. Whitney and P. Jacquod, Phys. Rev. Lett. 94 (2005) 116801.

[21] R. S. Whitney and P. Jacquod, Phys. Rev. Lett. 96 (2006) 206804.

[22] P. Vidal and E. Kanzieper, Phys. Rev. Lett. 108 (2012) 206806.

[23] P. Marconcini, M. Totaro, G. Basso and M. Macucci, AIP Advances 3 (2013) 062131. 
12 M. Macucci and P. Marconcini

[24] R. Landauer and T. Martin, Physica B 175 (1991) 167.

[25] A. van der Ziel, Noise in Solid State Devices and Circuits (John Wiley \& Sons, New York, 1986), pp. 18, 100

[26] G. B. Lesovik, Pis'ma Zh. Eksp. Teor. Fiz. 49 (1989) 515 [JETP Lett. 49 (1989) 594].

[27] M. Büttiker, Phys. Rev. Lett. 65 (1990) 2901.

[28] A. J. Makowski, P. Peplowski, S. T. Dembinski, "Chaotic causal trajectories: the role of the phase of stationary states," Physics Letters A 266, 241 (2000).

[29] F. Sols, M. Macucci, U. Ravaioli and K. Hess, J. Appl. Phys. 66 (1989) 3892.

[30] M. Macucci, A. Galick and U. Ravaioli, Phys. Rev. B 52 (1995) 5210.

[31] P. Marconcini, International Journal of Circuits, Systems and Signal Processing 7 (2013) 173.

[32] P. Jacquod and R. S. Whitney, Proceedings of the 4th International Conference on Unsolved Problems of Noise and Fluctuations. AIP Conf. Proc., Gallipoli, Italy, Vol. 800 (2005), p. 225, DOI: 10.1063/1.2138618.

[33] E. V. Sukhorukov and O. M. Bulashenko, Phys. Rev. Lett. 94 (2005) 116803.

[34] P. Marconcini, M. Macucci, D. Logoteta and M. Totaro, Fluct. Noise Lett. 11 (2012) 1240012 